\title{
Cellular modulation of polymeric device surfaces: promise of adult stem cells for neuro-prosthetics
}

\author{
Anja Richter ${ }^{1,2}$, Charli Kruse ${ }^{2}$, Andreas Moser ${ }^{3}$, Ulrich G. Hofmann ${ }^{4}$ and Sandra Danner ${ }^{2 *}$ \\ 1 Graduate School for Computing in Medicine and Life Sciences, University of Luebeck, Luebeck, Germany \\ ${ }^{2}$ Group of Cell Differentiation, Fraunhofer Research Institution for Marine Biotechnology, Fraunhofer Society, Luebeck, Germany \\ ${ }^{3}$ Department of Neurology, University of Luebeck, Luebeck, Germany \\ ${ }^{4}$ Institute for Signal Processing, University of Luebeck, Luebeck, Germany
}

Edited by:

Victor Pikov, Huntington Medical

Research Institutes, USA

Reviewed by:

Florin Popescu, Fraunhofer FIRST, Germany

Silvestro Micera, Scuola Superiore

Sant'Anna, Italy

${ }^{*}$ Correspondence:

Sandra Danner, Group of Cell

Differentiation, Fraunhofer Research Institution for Marine Biotechnology,

Paul-Ehrlich-Straße 1-3, 23562

Luebeck, Germany.

e-mail: sandra.danner@emb.

fraunhofer.de
Minimizing the foreign body response is seen as one critical research strategy for implants especially when designed for immune-privileged organs like the brain. The context of this work is to improve deep brain stimulating devices used in a consistently growing spectrum of psychomotor and psychiatric diseases mainly in form of stiff electrodes. Based on the compliance match hypothesis of biocompatibility we present another step forward using flexible implant materials covered with brain cell-mimicking layers. We covered two types of flexible polyimide films with glandular stem cells derived from pancreatic acini. Using real time-PCR and fluorescent immunocytochemistry we analyzed markers representing various cell types of all three germ layers and stemness. The results demonstrate an unchanged differentiation potential of the polyimide fixated cells as measured by mRNA and protein level. Additionally we developed a fibrinous hydrogel coating to protect them against shear forces upon eventual implantation. By repeating previous analysis and additional metabolism tests for all stages we corroborate the validity of this improvement. Consequently we assume that a stem cell-containing cover may provide a native, fully and actively integrating brain-mimicking interface to the neuropil.

Keywords: stem cell, polyimide, neural prosthesis, fibrin, surface modification, foreign body response

\section{INTRODUCTION}

Neuro-modulatory and neuro-prosthetic devices are increasingly developed for a number of neurological and psychiatric disorders in various targets of the central nervous system (Berney and Vingerhoets, 2005; Rasche et al., 2006; Larson, 2008; Benabid et al., 2009). One of the most prominent examples can be found in the field of deep brain stimulation (DBS) to treat psychomotor disorders like Parkinson's disease (PD) affecting 1\% of the population in the age group above 60 (Benabid et al., 2000a,b; Jankovic and Aguilar, 2008; Hemm and Wardell, 2010).

Deep brain stimulation is an invasive treatment and requires the insertion of a stimulating electrode into appropriate target areas of the patient's brain. Unfortunately, insertion of an artificial device into the body results in a complex immune response. This so-called foreign body response on deep brain stimulating electrodes results at least in the establishment of a glial scar tissue by reactive astrocytes (Moss et al., 2004; McConnell et al., 2009a). Sometimes, especially dense glial barriers may severely affect the electrical connection between electrode and tissue to be stimulated (Williams et al., 1999; McConnell et al., 2009b).

This effect becomes increasingly important with decreasing electrode size as approached in most recent DBS electrodes (Martens et al., 2010). One main cause for this adversive encapsulation is thought to be the mechanical compliance mismatch between any probe and brain tissue. Therefore several approaches are documented to develop flexible probes, floating with the brain's micro motion and thus reducing mechanical friction and inflammatory responses (Rousche et al., 2001).

In addition to biomechanical compatibility, the "material has to perform with an appropriate host response..." (Williams, 2008). Thus our goal of surface biocompatibility is to minimize the activation of tissue-resident immunocompetent cells, as microglia and astrocytes, otherwise resulting in a bold glial scar (Polikov et al., 2005; Seymour and Kipke, 2007; Leach et al., 2010).

Documented efforts to overcome these circumstance includes coupling of proteins, neurotrophic peptides (reviewed in Rao and Winter, 2009), or anti-inflammatory drugs (Spataro et al., 2005) on the surface of polymeric and flexible probes. We propose a complete, beneficial cellular sheath covering the DBS electrodes to promote the active, less-immunogenic integration of a polymeric electrode into the brain tissue. For this purpose, neuronally differentiating adult stem cells seem to be the cell cover of choice.

Glandular adult stem cells isolated from pancreas, salivary glands, or sweat glands have been shown to exhibit improved growth characteristics and show multi-lineage differentiation into cell types of the three embryonic germ layers (Kruse et al., 2006; Rotter et al., 2008; Gorjup et al., 2009; Petschnik et al., 2009). In animal studies on skin regeneration these cells have an enormous benefit for full thickness skin wound healing where they accelerated wound closure and improved vascularization (Egana et al., 2009; Salem et al., 2009). Pancreatic stem cells (PSCs) have the ability to differentiate in vitro spontaneously into neural cell 
types (Kruse et al., 2006; Gorjup et al., 2009; Rapoport et al., 2009a,b). Adult stem cells with neuronal differentiation potential are therefore a promising tool to challenge the formation of the glial scar tissue by presenting the brain's native neuronal cells on the polymeric device surface.

To protect the sensible cellular layer on the device during the implantation procedure we searched for a biodegradable, easy applicable, mostly native protecting coverage of it. The literature offers possibilities with well studied hydrogels that enable a jelly like protection while attracting neurons. Prominent examples are compositions based upon collagen, agarose, chitosan, or fibrin (Zhong and Bellamkonda, 2008). Further improvement of hydrogels, e.g., by additive extracellular matrix molecules like laminin to reduce astrogliosis was shown as well (Yu et al., 1999; He et al., 2006).

We tested fibrin-hydrogels as they are highly definable, easily applicable, and clinically approved. Fibrin is a hydrogel of crosslinked fibronectin-fibers produced naturally in the body. It combines a favorable attraction of axons with neuro-supportive effects in damaged neural tissues by reducing astroglial response (Itosaka et al., 2009; Johnson et al., 2009a,b).

Here we show that the differentiation potential of the favored cells is maintained even if they are grown on a flexible polymeric, here polyimide, surface. Since electrode insertion leads to mechanical abrasion forces, we investigated the influence of a possible protective fibrin-based hydrogel on the cells.

\section{MATERIALS AND METHODS}

\section{ISOLATION, CULTIVATION, AND STORAGE OF PANCREATIC STEM CELLS}

Pancreatic stem cells were isolated from male Wistar rats (age between 8 and 12 weeks) according to the protocol of Kruse et al. (2006). Briefly, the pancreas was removed from the animal body and attached fat, lymph nodes, and blood vessels were dissected. Subsequently the digestion of the pancreatic tissue was performed with HEPES-Eagle medium ( $\mathrm{pH} 7.4$ ), 0.1 mM HEPESbuffer (pH 7.6, Carl Roth GmbH + Co. KG, Karlsruhe, Germany), $70 \%$ (v/v) MEM (Biochrom AG, Berlin, Germany), 0.5\% (v/v) Trasylol (Bayer AG, Leverkusen, Germany), 1\% (w/v) bovine serum albumin (PAA Laboratories GmbH, Pasching, Austria), $2.4 \mathrm{mM}$ $\mathrm{CaCl}_{2}$ (Carl Roth $\mathrm{GmbH}+\mathrm{Co} . \mathrm{KG}$, Karlsruhe, Germany), and collagenase (0.63 PZ/mg, Serva, Heidelberg, Germany). The following suction with different glass pipettes and filtration through a nylon mesh resulted in an efficient dissociation of the pancreatic acini. After centrifugation at $700 \mathrm{rpm}$ for $5 \mathrm{~min}$ the digestion media was removed and the pellet was washed two times by resuspending it in Dulbecco's modified Eagle's medium (DMEM, Gibco Invitrogen, Darmstadt, Germany) supplemented with $20 \%$ fetal calf serum (FCS, PAA Laboratories GmbH, Pasching, Austria) and antibiotics and repeating the centrifugation step. Finally the isolated acini were resuspended in DMEM and cultured in a $25 \mathrm{~cm}^{2}$ flask (TPP Techno Plastic Products AG, Trasadingen, Suisse) at $37^{\circ} \mathrm{C}$ in a $5 \% \mathrm{CO}_{2}$ humidified atmosphere. Two days post preparation the medium was changed and adherent acini with outgrowing spindle-shaped cells were visible. When reaching confluence the PSCs were trypsinized ( $0.1 \%$ trypsin, PAA Laboratories $\mathrm{GmbH}$, Pasching, Austria) and subcultured in a ratio of 1:3. The following cultivation was performed with DMEM and 10\% FCS $(\mathrm{v} / \mathrm{v})$ supplemented with penicillin $(100 \mathrm{U} / \mathrm{ml})$ and streptomycin $(0.1 \mathrm{mg} / \mathrm{ml}$; both PAA Laboratories GmbH, Pasching, Austria). To generate sufficient amounts of cells the culture was propagated for several passages and two third of cells were stored at each passage by cryopreservation using 90\% FCS with 10\% dimethylsulfoxide (DMSO, Sigma-Aldrich Biochemie GmbH, Hamburg, Germany).

\section{POLYIMIDE SAMPLE PRODUCTION}

Since the composition of polyimide may have an impact on our cells, we tested polyimide films from two sources: the Institut für Mikrotechnik Mainz GmbH (IMM) used DuPont PI 2731 (DuPont, Wilmington, NC, USA) and the Fraunhofer Institute for Silicon Technology (ISIT) used HD MicroSystems PI-2555 (HD MicroSystem, Hitachi Chemical, DuPont, Wilmington, NC, USA).

Polyimide processing has been performed following the standard procedures according to manufacturer's instructions. First, a thin polymer layer is deposited on a $5^{\prime \prime}$ (IMM) or $6^{\prime \prime}$ (ISIT) microelectronic grade silicon wafer serving as substrate. The primary interface layer provides on one hand sufficient adhesion for later lithographical processing and allows on the other hand easy, damage-free peeling of the final devices from the carrier substrate. Hereafter, a $10-20 \mu \mathrm{m}$ thick layer of the PI material DuPont PI 2731 (IMM) or HD Microsystems PI-2555 (ISIT) is spin coated. Spun material is dried on a $90^{\circ} \mathrm{C}$ hot plate and cured for an hour at $160-200^{\circ} \mathrm{C}$.

Actual sample production was done by cleaning and disinfecting the PI film with ethanol 70\% (Carl Roth $\mathrm{GmbH}+$ Co. KG, Karlsruhe, Germany) and cutting with a surgical scalpel and a polystyrene mask into pieces.

\section{CELL SEEDING ON POLYIMIDE FILMS}

Polyimide film was cut directly on the production wafer into suitable pieces to glue them onto the bottom of the cell culture plastic. For immunocytochemistry, pieces had a size of $1 \mathrm{~cm} \times 1.5 \mathrm{~cm}$ and were glued with high vacuum silicone grease (Dow Corning, Midland, TX, USA) into culture slides (BD, Heidelberg, Germany). For (RT)-PCR a circle with a diameter of $6 \mathrm{~cm}$ was glued with silicone into a $60 \mathrm{~mm}$ Petri dish (TPP Techno Plastic Products AG, Trasadingen, Suisse). According to the subculture ratio cells were seeded with a density of approximately 20000 cells per $\mathrm{cm}^{2}$ using DMEM with $10 \%$ FCS and antibiotics. After reaching a confluence of more than $80 \%$ the cells were used for subsequent described analysis.

\section{GROWTH ANALYSIS}

For growth analysis cells were trypsinized as usual for passaging and counted with a Nucleocounter (ChemoMetec A/S, Allerød, Denmark) using the corresponding Lysis-Buffer and StabilizationBuffer (both ChemoMetec A/S, Allerød, Denmark). Starting with a seeding density of 5000 cells per $\mathrm{cm}^{2}$ two replicates for each measurement point were seeded into two wells of a 24 -well plate (TPP Techno Plastic Products AG, Trasadingen, Suisse) using above described normal cultivation media. For analyzing the growth rate on polyimide membranes, pieces of $1 \mathrm{~cm} \times 1 \mathrm{~cm}$ are glued with silicone grease on the bottom of a well. At day 1, 2, 3, 4, 7, and 8 after 
seeding the cell number per $\mathrm{cm}^{2}$ growth area was determined by lysing the cells directly inside the well or extracting the polyimide piece and lyse the cells on it separately in a reaction tube. The nuclei were counted with the Nucleocounter. Each measuring point was evaluated by the average of triple repeats.

\section{IMMUNOCYTOCHEMISTRY ANALYSIS}

Cultivated cells in the culture slides were washed with PBS (Gibco Invitrogen, Darmstadt, Germany) and fixed with methanol/acetone (7:3, both Carl Roth GmbH + Co. KG, Karlsruhe, Germany) and $1 \mathrm{mg} / \mathrm{ml}$ DAPI (Roche Deutschland Holding $\mathrm{GmbH}$, Grenzach-Wyhlen, Germany) for $5 \mathrm{~min}$ at $20^{\circ} \mathrm{C}$. Then the samples were washed three times with PBS and blocked with $16.5 \mu \mathrm{l} / \mathrm{ml}$ normal goat serum (Vector Laboratories Ltd., Peterborough, UK) in PBS for $20 \mathrm{~min}$ at $20^{\circ} \mathrm{C}$. After removing the blocking mixture primary antibodies diluted in TBST with $1 \%(\mathrm{w} / \mathrm{v}) \mathrm{BSA}$ were added and incubated for $1 \mathrm{~h}$ at $37^{\circ} \mathrm{C}$ in a humidified chamber. Used primary antibodies for neuronal differentiation are raised against glial fibrillary acidic protein (rabbit polyclonal antiGFAP, Dako Deutschland GmbH, Hamburg, Germany, 1:100), neurofilament (1:1:1 mixture of antibodies rabbit polyclonal antineurofilament light, medium, heavy chain, AbD Serotec a division of MorphoSys AG, Martinsried/Planegg, Germany, 1:500), nestin (mouse monoclonal anti-nestin, Chemicon, Hofheim, Germany, 1:200), and for mesodermal differentiation against alpha smooth actin (mouse monoclonal anti-smooth muscle actin, Dako Deutschland GmbH, Hamburg, Germany, 1:100). Prior to incubation with the secondary antibodies at the same conditions as used for the primary antibodies all samples are rinsed three times with PBS. For detection of primary antibodies Cy3-labeled goat-anti-mouse IgG (Dianova, Hamburg, Germany, 1:400) and FITC-labeled goat-anti-rabbit IgG (Dianova, Hamburg, Germany, 1:200) were diluted in PBS. A threefold washing step with PBS, short equilibration with bidest. $\mathrm{H}_{2} \mathrm{O}$ and mounting the samples with Vectashield mounting medium (Vector Laboratories Ltd., Peterborough, UK) finalized the procedure.

\section{PCR ANALYSIS}

Cells grown in Petri dishes were washed once with PBS and then scraped with a cell scraper (TPP Techno Plastic Products AG, Trasadingen, Suisse) in $2 \mathrm{ml}$ PBS. The cells were pelleted and stored at $-80^{\circ} \mathrm{C}$ until the isolation of total RNA using a RNeasy Plus Mini Kit and the QIAcube instrument (both QIAGEN GmbH, Hilden, Germany) for automated RNA isolation according to manufacturer protocols. The concentration of total RNA was measured with a NanoDrop Spectrophotometer ND-1000 (Peqlab, Erlangen, Germany) and $1 \mu \mathrm{g}$ of total RNA was used for reverse transcription with the QuantiTect reverse transcription kit (QIAGEN GmbH, Hilden, Germany) including a genomic DNA digestion step. The following polymerase chain reaction was performed three fold as duplicates with $1 \mu \mathrm{l} \mathrm{cDNA}$ within a $25 \mu \mathrm{l}$ reaction volume of QuantiFast SYBR Green PCR kit (QIAGEN GmbH, Hilden, Germany) using a RealPlex2 (Eppendorf AG, Hamburg, Germany). Primers are all commercially available and validated by QIAGEN (Table 1). The generated amplicons were visualized using the capillary gel electrophoresis system QIAxcel (QIAGEN GmbH, Hilden, Germany).
Table 1 | List of used QuantiTect primer assays.

\begin{tabular}{lll}
$\begin{array}{l}\text { Gene } \\
\text { symbol }\end{array}$ & Assay name & $\begin{array}{l}\text { Catalog } \\
\text { number }\end{array}$ \\
\hline Act b & Rn_Actb_1_SG QuantiTect primer assay (200) & OT00193473 \\
Acta2 & Rn_Acta2_1_SG QuantiTect primer assay (200) & OT01615901 \\
Pou5f1 & Rn_Pou5f1_1_SG QuantiTect primer assay (200) & OT00455028 \\
CD9 & Rn_Cd9_2_SG QuantiTect primer assay (200) & OT01566810 \\
Gata4 & Rn_Gata4_1_SG QuantiTect primer assay (200) & OT01082207 \\
Pparg & Rn_Pparg_1_SG QuantiTect primer assay (200) & OT00186172 \\
Gfap & Rn_Gfap_1_SG QuantiTect primer assay (200) & OT00195517 \\
Nes & Rn_Nes_1_SG QuantiTect primer assay (200) & QT00376922 \\
Nef3 & Rn_Nef3_1_SG QuantiTect primer assay (200) & OT00180572 \\
Tubb3 & Rn_Tubb3_1_SG QuantiTect primer assay (200) & OT00188818 \\
Eno2 & Rn_Eno2_1_SG QuantiTect primer assay (200) & OT00179837 \\
\hline
\end{tabular}

\section{FIBRIN COVER}

When confluence on polyimide was reached we covered the cells with fibrin by dip-coating and used them for shear force experiments (Richter et al., 2010) or further cultivation and analyses. Polyimide films with cells were dipped sequentially in culture medium with human plasma fibrinogen $(1.5 \mathrm{mg} / \mathrm{ml}$; Calbiochem by Merck KGaA, Darmstadt, Germany) and then with human plasma thrombin $(0.1 \mathrm{unit} / \mathrm{ml}$, Calbiochem by Merck KGaA, Darmstadt, Germany). After immediate return to the incubator for a 5-min polymerization step the samples were used for shear force experiments or again immersed in culture medium for ongoing cultivation. After 7 days of culture fibrin-covered specimens were used for RT-PCR and protein analyses. For all other exminations, e.g., growth curve, metabolism analysis, shear stress test, the cultivation period was adapted to the corresponding test.

\section{METABOLISM ANALYSIS}

Measurement of dextrose and lactate in the medium was performed in parallel to the growth curve set up by measuring of $50 \mu \mathrm{l}$ medium with the Biochemistry Analyzer 2700 Select [YSI (UK) Limited, Fleet, UK].

\section{SHEAR STRESS TESTS}

The shear stress tests were performed using a piezo-driven micromanipulator (Eppendorf, Hamburg, Germany). The complete setting is described elsewhere (Richter et al., 2010). Briefly we connected a surgical clamp to the micromanipulator arm under an angle of $45^{\circ}$ to the bottom plate. Then a Petri dish was positioned under the arm and the lower half of a brain dummy made of $2 \%(\mathrm{w} / \mathrm{v})$ low melt agarose in PBS was positioned inside the dish under the arm. The total size of the dummy was tailored to the sizes of a rat brain by cutting it with a scalpel out of the gross matrix. Then the dish was filled with PBS until the lower half of the dummy was totally covered. After repositioning of it the polyimide-strip was placed on the dummy and the identical upper half of the dummy was carefully positioned on the sample. An overlapping end of the polyimide-strip was connected to the surgical clamp and the horizontal movement of the micromanipulator arm followed with constant $7500 \mu \mathrm{m}$ per second. Thereby shear stress could be applied in an automatized, reproducible, and 
standardized procedure. The whole experiment was performed as well with rat brain hemispheres using the same setting.

\section{RESULTS}

\section{GROWTH CHARACTERISTICS ON POLYIMIDE SURFACES}

To determine influences of polyimide surfaces on cell growth properties we performed a growth analysis. Proliferation of the pancreatic stem cell population was monitored on the two chosen polyimides DuPont PI 2731 (termed ${ }^{\circ} 1$ in the following) or HD Microsystems PI-2555 ( ${ }^{\circ} 2$ ) with an initial amount of 5000 cells per $\mathrm{cm}^{2}$. Figure 1 shows a DIC micrograph with attached cells on polyimide ${ }^{\circ} 2$ as well as on cell culture plastic indicating a good acceptance of polyimide as growth substrate.

Proliferation of cells on the different materials was characterized by growth curves over a period of 8 days. Figure 2 depicts comparable growth curves on PI and cell culture plastic during passage 9.

The growth of the multipotent pancreatic stem cells was not significantly changed on both polyimide films compared to cell culture plastic used for standard cultivation (Figure 2).

Despite the differing curve shapes during early cultivation phase of these cells the general proliferation behavior was not influenced by neither polyimide film compared to the corresponding control. Full confluence, meaning a complete coverage of the test surface by cells, was reached at the same point in time (after 4 days). Apparently, both analyzed polyimides exhibited no adverse effects on cellular attachment and proliferation.

\section{MAINTENANCE OF STEM CELL PROPERTIES}

The chemical and physical properties of the surface may actively influence the performance of stem cells (Engler et al., 2006). Given this fact we have to ensure that the polyimide surface itself does not change the pancreatic stem cell's potential for differentiation.

To determine the cells differentiation behavior we compared gene and protein expression of PSCs grown on polyimide and normal cell culture surface. An immunocytochemistry analysis and a reverse transcriptase (RT)-PCR was performed for stem cell markers and neuronal markers. Clearly, for possible implantation of cell-covered electrodes in the brain, a neural lineage is the differentiation lineage of interest (Figure 3).

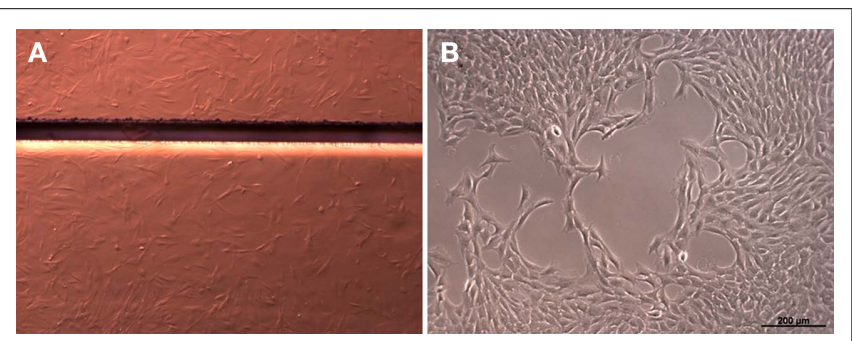

FIGURE 1 | Cells grown on polyimide (A) and on standard cell culture plastic (B). The light micrograph shows cells as irregular structures on the polyimide films. The horizontal bar shows a boundary between two polyimide pieces (Magnification 6x, Discovery V.8; Carl Zeiss GmbH, Oberkochen, Germany). For normal cell culture plastic the cells are depicted by phase-contrast (Axiovert 40C, Carl Zeiss GmbH, Oberkochen, Germany).
To verify the spontaneous differentiation potential of PSC on the substrates we evaluated several transcripts indicating stemness (nestin, CD9, oct4, klf4, c-myc), mesodermal differentiation potential (PPAR $\gamma$, SPP1, alpha smooth muscle actin), and endodermal differentiation potential (GATA4) as well as several transcripts for neural differentiation (neurofilaments, GFAP, S100, MBP, Tubulin $\beta 3$, enolase, NCAM; Figure 3). To ensure expression of selected proteins corresponding to the transcripts we performed immunocytochemical staining for nestin, neurofilaments, GFAP, and smooth muscle actin alpha (Figure 4).

As nestin is considered a general adult stem cell marker and indicates ectodermal differentiation potential of stem or precursor cells, we were able to corroborate the stem cell status of the cells when grown on polyimide (Wiese et al., 2004). The detection of neurofilaments at translational and transcriptional levels indicates the maintenance of the neural differentiation potential. The same is true for GFAP, as we found the protein expressed although the transcript was not detected by our PCR approach. This may be due to a short molecule lifetime of the GFAP mRNA.

The expression of alpha smooth muscle actin, a myogenic marker, reveals the maintenance of the mesodermal differentiation potential indicative for the multi-lineage potential of the cells. Qualitatively both polyimides enable our cells to differentiate spontaneously the same way as they do on cell culture plastic.

As qualitatively no changes in the differentiation potential became obvious we focused our decision between the two polyimides for further use on the growth behavior. The higher similarity of the growth curves between plastic and polyimide 1 over polyimide 2 let to the choice to use this for further investigations.

\section{PROTECTION OF THE CELL SHEATH AGAINST SHEAR STRESS - CHARACTERIZATION OF FIBRIN LAYER COVERING}

During insertion of cell-covered probes in brain tissue or comparable material models, the penetrated tissue will exert shear stress on the cells. Our previous work showed a standardized method to simulate these effects (Richter et al., 2010). In order to protect the sensitive cell layer against this stress, we introduced a hydrogel covering of cells by fibrin dip-coating (Johnson et al., 2009a,b; Figure 5).

To characterize cells' behavior under this coverage, e.g., to exclude that cells migrate into the fibrin-matrix and are lost to the confluent layer on the probe, we coated our devices at an almost confluent stadium with fibrin. In consequence we were not able to perform a growth curve on polyimide with fibrin as we did before. Instead the proliferative activity was examined by immunocytochemistry using an antibody against Ki67, considered a proliferation marker (Gerdes et al., 1983). Despite the nearly tight cell population we found several cells displaying a positive staining for Ki67 (Figure 6).

As mentioned earlier changes of the cells' surrounding matrix may influence their general behavior. Thus we analyzed the differentiation potential of the stem cells under fibrin coating. No qualitative changes in the transcriptome were found by RT-PCR, when cells were cultured with the fibrin layer (Figure 7).

The expression of all genes resembles the situation without fibrin. GFAP expression remains abundant and other indicators for stemness and differentiation lineages are expressed in similar 


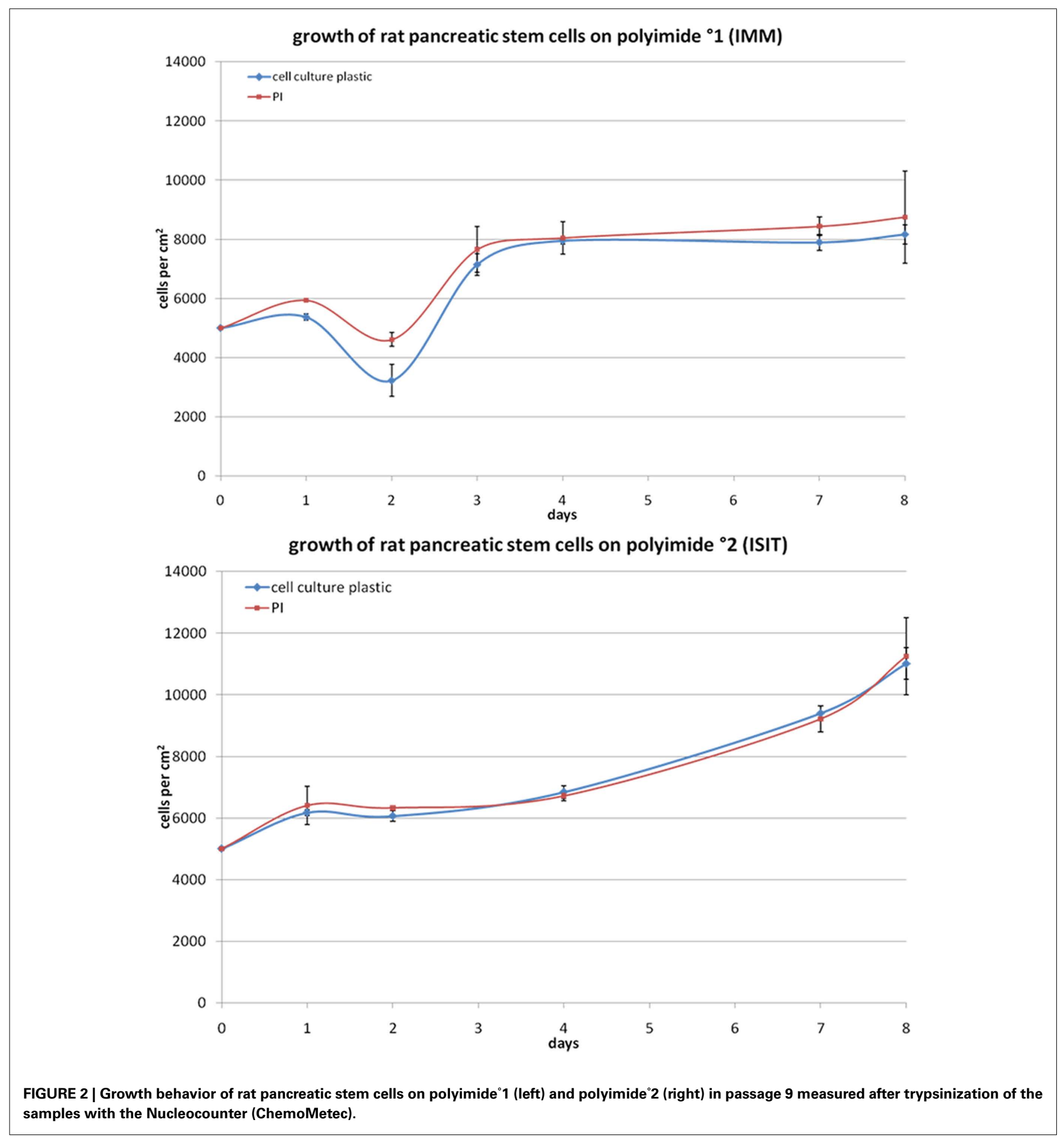

intensity as we have seen before. Qualitatively, the gene expression pattern does not change indicating a good tolerance of our cells when cultured with fibrin.

Immunocytochemical staining of cells covered with a fibrin layer confirmed maintained cell characteristics as suggested by RTPCR. All considered proteins (smooth muscle actin, GFAP, nestin, neurofilaments) were successfully stained (Figure 8).

Smooth muscle actin alpha was found in a greater number of cells. We hypothesize that this may be caused by the change in surrounding matrix' elasticity. Cells might have tried to attach to the fibrin-matrix in all three dimensions resulting in a stronger expression of actin filaments to deal with surrounding elastic forces.

\section{COMPARATIVE METABOLISM ANALYSES}

As mentioned above we covered the cells at an almost confluent stadium of the cultivation. We circumvented the analytical penalty concerning the cells proliferation status with a Ki67 


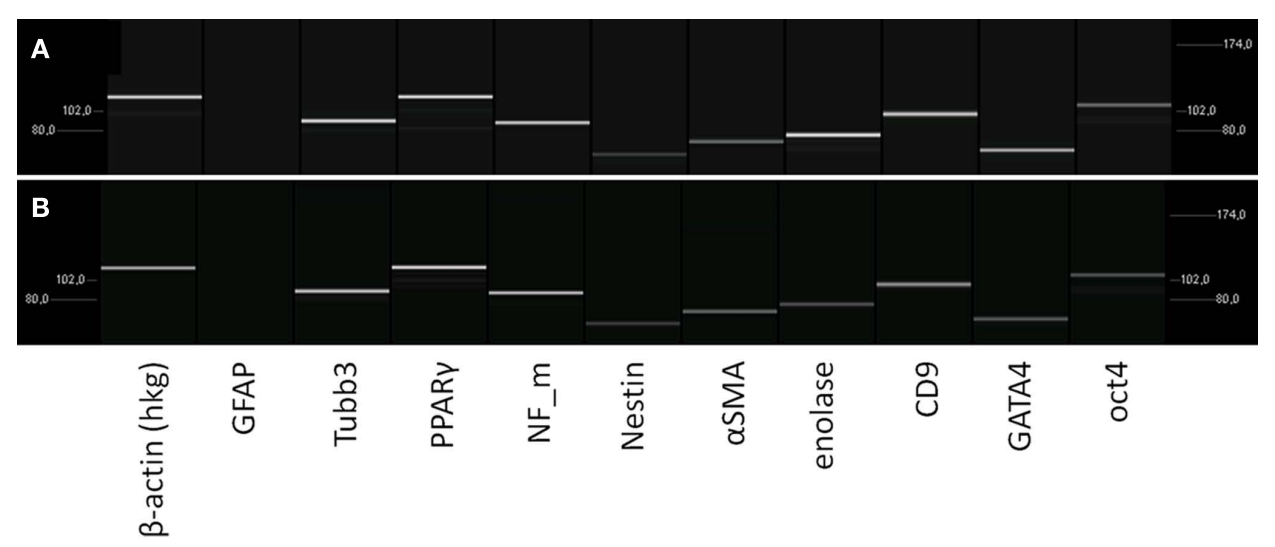

FIGURE 3 | Characterization of the mRNA-expression in rat pancreatic stem cells grown on cell culture plastic (A) and polyimide $\mathbf{2}$ film (B). Cells were analyzed in regard to their transcriptome by utilizing RT-PCR. The subsequent capillary gel electrophoresis shows transcripts typical for all germ layers and stemness.

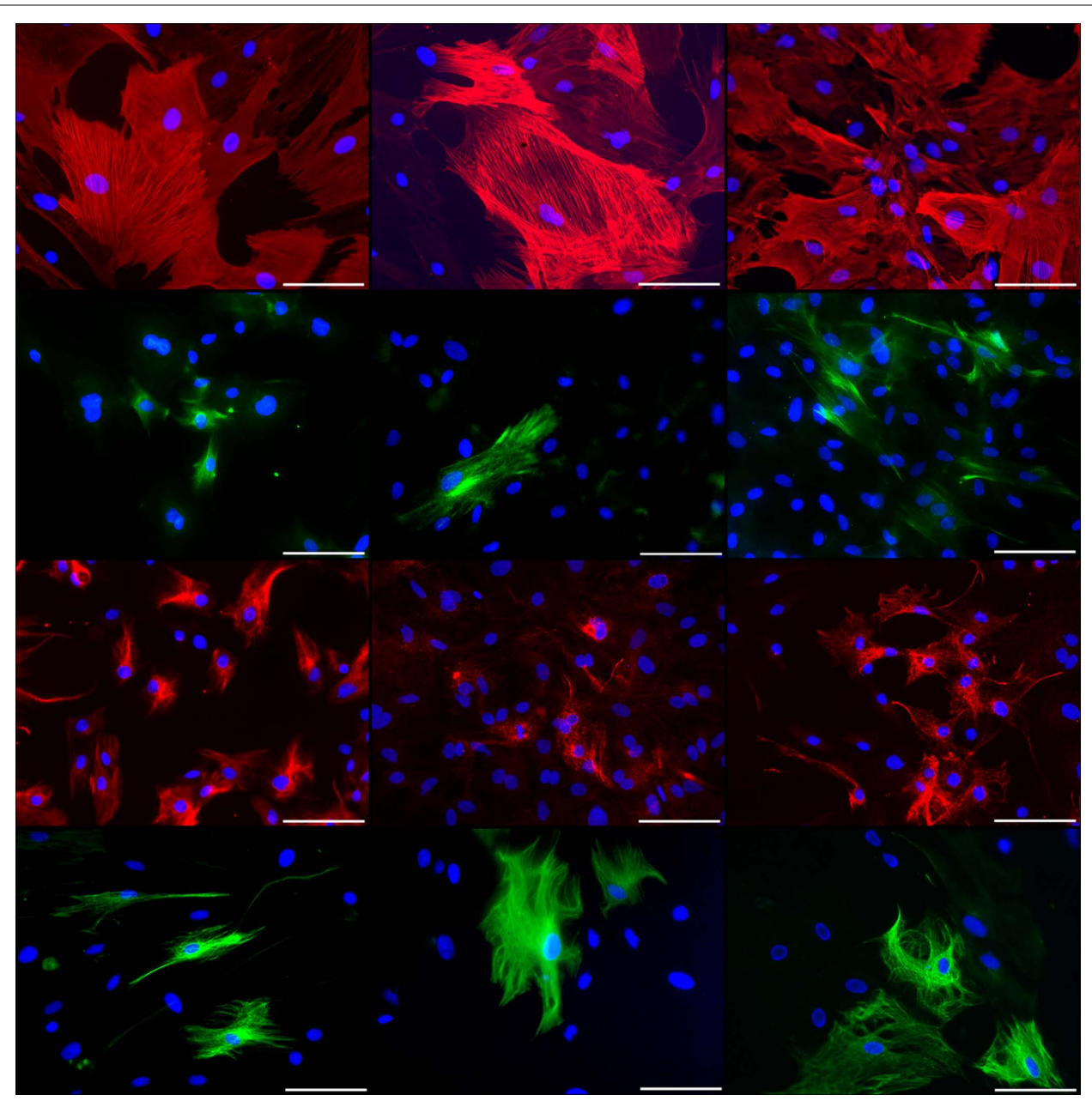

FIGURE 4 | Immunocytochemical staining of rat pancreatic stem cells grown on cell culture plastic (left column), polyimide 1 from IMM (center column), and polyimide 2 from ISIT (right column). Cells are stained for alpha smooth muscle actin (SMA, first row), glial fibrillary acidic protein (GFAP, second row), nestin (third row), and neurofilaments (fourth row). Secondary antibody in green was FITC-labeled goat-anti-rabbit and in red was Cy3-labeled goat-anti-mouse. The nuclear counterstaining was made with DAPI. All scale bars represent $100 \mu \mathrm{m}$. Images are made with an Axioskop2 or Axio Observer.Z1 (both Carl Zeiss GmbH, Oberkochen, Germany). 
staining and still found proliferating cells in the population. To undergird the cells fitness we analyzed the cells metabolism by a comparative dextrose-lactate-measurement for 7 days (Figure 9).

During the cultivation the population consumes dextrose from the medium and produces lactate. We found at all cultivation conditions a continuous decrease in concentration of dextrose and vice versa an increase for the concentration of lactate. The slightly raised concentration of both for the blanks (medium only) is due to evaporation. Cells with a fibrin cover displayed a minimally reduced metabolism. That may be a result of a hindered exchange

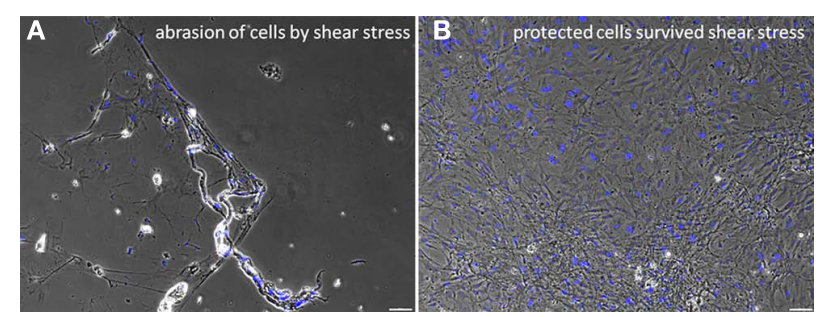

FIGURE 5 | Effects of shear stress examined by an abrasion experiment. Strong abrasion of the confluent cell layer due to the exerted pulling forces is visible (A). This was prevented by applying the protecting fibrin cover (B). The black dots in the right picture indicate air bubbles in the fibrin gel above the cells. Scale bars represent $100 \mu \mathrm{m}$ (Axio Observer.Z1, Carl Zeiss GmbH, Oberkochen, Germany).

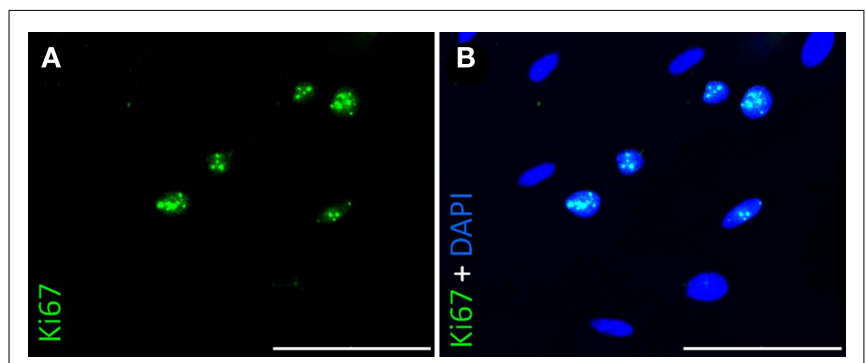

FIGURE 6 | Immunocytochemical staining of rat pancreatic stem cells for Ki67 grown on polyimide $\mathbf{2}$ from ISIT. Nuclei were positively stained for Ki67 (A) and showed co-localisation with nuclear counterstain DAPI (B). Scale bars represent $100 \mu \mathrm{m}$ (Axio Observer.Z1, Carl Zeiss GmbH, Oberkochen, Germany). of dextrose and lactate by the hydrogel mesh since we performed the measurements with the supernatant medium. In general we found for all conditions the same metabolism profile corroborating good suitability of the cellular cover of polyimide with a protecting fibrin gel.

\section{DISCUSSION}

The invasive treatment with DBS electrodes is a promising clinical application for neurological disorders. For a substantial percentage of psychomotor and psychiatric inabilities novel neuro-prosthetic devices offer potent future therapies. Some issues have to be taken into account regarding the device material, architecture, and mechanics. We believe that the usage of flexible electrodes will improve the long-term success of this therapy, since they have the ability to float with the brain's motion. Our goal was to enhance the quality of a potential therapy by combining a flexible polyimide probe with a living stem cell surface. This should furthermore enhance the biocompatibility, integrity, and long-term functionality of the device in the recipient's brain. In particular, mimicking host's own implant surface may help to minimize the enduring activation of immune cells resulting in an implant surrounding glial scar tissue. We present here the behavior of adult glandular stem cells on different polyimide substrates in vitro and pave the way for their application as probes in vivo.

To our knowledge we show for the first time that adult stem cells attach to, proliferate on and keep their potential on polyimide surfaces. We document that two different polyimides were equally well accepted as growth surfaces by the cells. The glandular stem cells swiftly created a confluent cell population without changing their composition compared to normal cell culture plastic. PSCs differentiate spontaneously in vitro into cells derived from all embryonic germ layers. The expression of several markers for stemness, ectoderm, mesoderm, and endoderm was proven successfully on transcriptional and translational level. This multi-lineage potential was maintained when cells grew on polyimide.

Regarding a possible application for DBS we focused especially on the neuronal differentiation potential of the cells. This will open up the possibility for a better integration of devices by connecting brain and device through neuronally differentiating stem cells. Comparing the expression of neural-related mRNAs and proteins of cells cultivated on cell culture plastic and polyimide, we

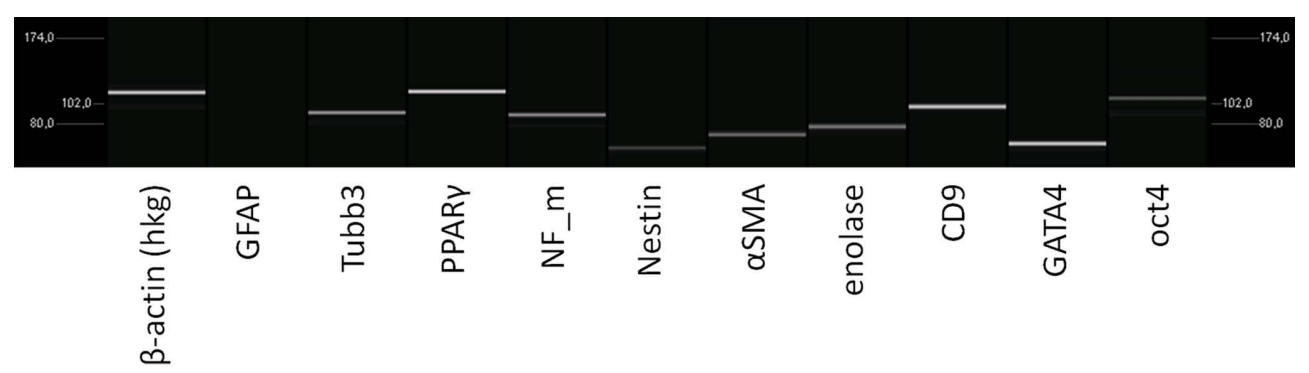

FIGURE 7 | Analysis of the transcriptome of rat pancreatic stem cells grown on polyimide $\mathbf{2}$ film covered with fibrin. The considered markers are assigned below. 
examined a strong differentiation potential for both conditions independent from the type of polyimide.

Previous work from Purcell et al. (2009) confirmed that cellseeded cortical probes have a beneficial effect on the early brain tissue response after implantation. They fabricated parylene devices,

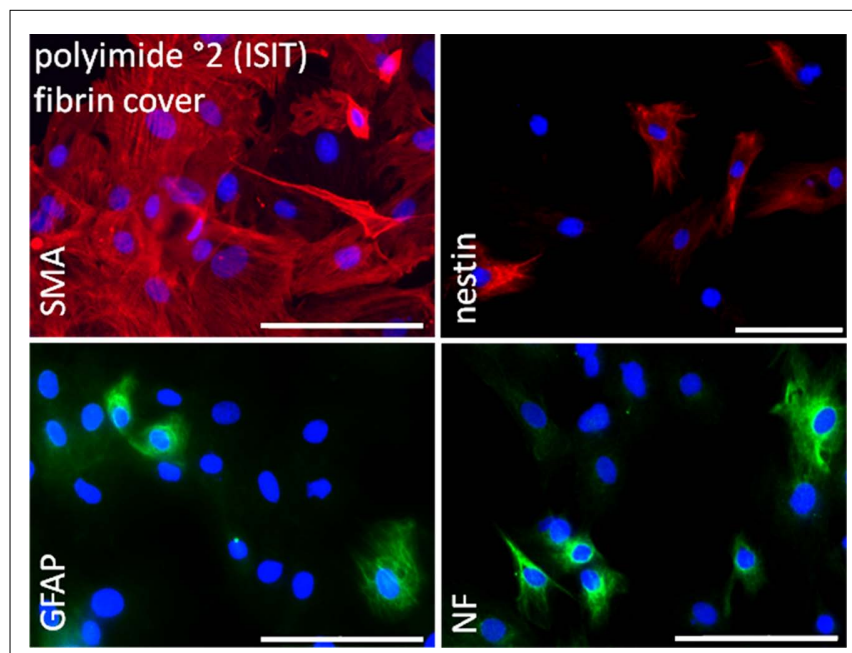

FIGURE 8 | Immunocytochemical analysis of rat pancreatic stem cells grown on polyimide $\mathbf{2}$ film covered with fibrin. Cells are stained for alpha smooth muscle actin (SMA), glial fibrillary acidic protein (GFAP), nestin, and neurofilaments (NF). Secondary antibody in green was FITC-labeled goat-anti-rabbit and in red was Cy3-labeled goat-anti-mouse. The nuclear counterstaining was made with DAPI. Scale bars represent $100 \mu \mathrm{m}$ (Axio Observer.Z1, Carl Zeiss GmbH, Oberkochen, Germany). which were built-up by an open well seeded with mouse cortical neural stem cells encapsulated in an alginate hydrogel scaffold (Purcell et al., 2009). The study, carried out in a rat model, described an improved healing process over a period of 3 month post-implantation. This promising result encouraged us to test this idea with a syngeneic source of cells on flexible devices. This is the best approximation to test cell-seeded neuro-prostheses in small mammalian models for potential autologous therapy strategies in humans.

With respect to the development of effective and applicable clinical approaches we had to ensure attachment and survival of the cell sheath during the implantation process. We addressed this problem in a previous work and simulated shear forces with a standardized automated technology (Richter et al., 2010). The results clearly showed that the cell layer was peeled off by the implantation's shear forces, but we overcame this impediment by protecting the cell sheath with a fibrin-based hydrogel. Several approaches using fibrin as three-dimensional matrix for cell delivery to the central nervous system had a beneficial outcome. Transplanting bone marrow stromal cells into the spinal cord or the cortex of rats effects in enhanced survival, migration, and differentiation of the transplanted cells by using fibrin-matrix constructs (Itosaka et al., 2009; Yasuda et al., 2009). In addition fibrin gels themselves have neurite growth promoting and astrocyte repellent effects, which is of crucial interest for a successful electrophysiological connection to the DBS devices (Johnson et al., 2009a,b). Whether a further improvement of the fibrinous protection layer by addition of other extracellular matrix proteins is beneficial will be of interest when first in vivo results are acquired and compared with the literature.

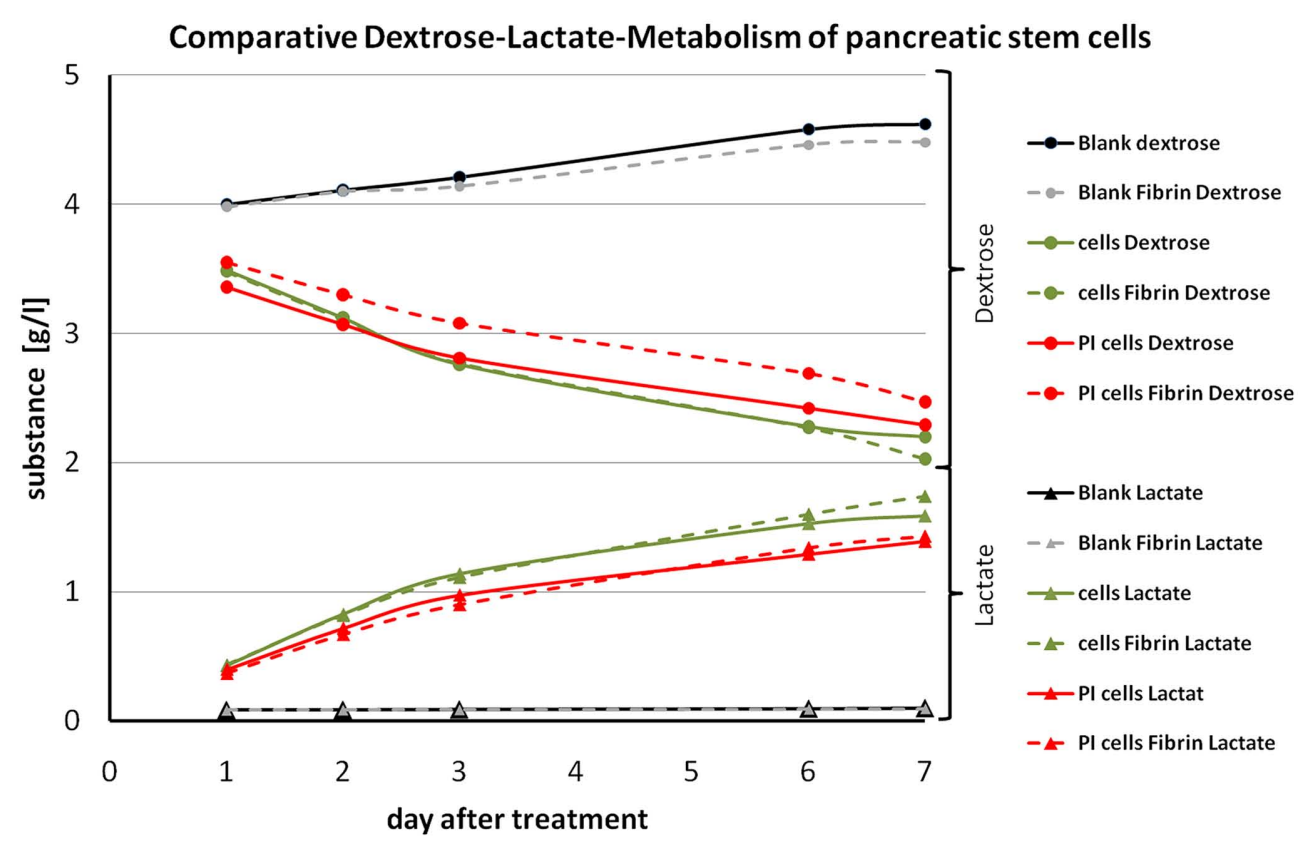

FIGURE 9 | Graph of the comparative dextrose-lactate metabolism of pancreatic stem cells. Dextrose curves for all samples are indicated by a dot at each measurement and lactate curves are indicated by a triangle at each measurement. Dashed lines are used for fibrin samples and solid lines for non-coated samples. Gray and black lines represent the blanks. Green lines are used for samples on cell culture plastic and red lines for samples on polyimide films. Sample names are depicted in the legend. 
Furthermore we pursued the analysis of the stem cell status of our cells under fibrin coverage. Our results showed that fibrin is conducive to protect the cell layer and has no impact on the proliferation, metabolism, stemness, and differentiation potential of pancreatic stem cells.

Our work is in line with previous matrix-based transplantation studies on skin regeneration with pancreatic stem cells (Egana et al., 2009; Salem et al., 2009). A faster wound closure coupled with an increased vascularization and improved morphology was documented in these previous studies mediated by pancreatic stem cells applied within a scaffold. The pancreatic stem cells we used in our experiments are best characterized (Kruse et al., 2006). Thus we use them as a representative for easier accessible glandular-derived stem cell populations from submandibular or sweat glands that are currently under investigation (Gorjup et al., 2009; Petschnik et al., 2009).

We can only hypothesize on the effects of the stem cell or fibrinous cover on the electrode-tissue impedance, which is of key interest in ongoing studies. Nevertheless we expect no gross impedance increase of a single-layer stem cell coverage with a thickness of approximately $20 \mu \mathrm{m}$, which falls below the critical thickness of $50 \mu \mathrm{m}$ (McConnell et al., 2009b; Mercanzini et al., 2009). When compared to astrogliotic scar tissue, we did not produce such a tightly woven cell layer which in addition produces an isolating extracellular matrix. With respect to the hydrogel an estimation concerning the impedance cannot be done easily. We

\section{REFERENCES}

Benabid, A. L., Chabardes, S., Mitrofanis, J., and Pollak, P. (2009). Deep brain stimulation of the subthalamic nucleus for the treatment of Parkinson's disease. Lancet Neurol. 8, 67-81.

Benabid, A. L., Koudsie, A., Benazzouz, A., Fraix, V., Ashraf, A., Le Bas, J. F., Chabardes, S., and Pollak, P. (2000a). Subthalamic stimulation for Parkinson's disease. Arch. Med. Res. 31, 282-289.

Benabid, A. L., Koudsie, A., Pollak, P., Kahane, P., Chabardes, S., Hirsch, E., Marescaux, C., and Benazzouz, A. (2000b). Future prospects of brain stimulation. Neurol. Res. 22, 237-246.

Berney, A., and Vingerhoets, F. (2005). Novel brain stimulation techniques: therapeutic perspectives in psychiatry. Rev. Med. Suisse 1, 2162-2164, 2166.

Egana, J. T., Danner, S., Kremer, M., Rapoport, D. H., Lohmeyer, J. A., Dye, J. F., Hopfner, U., Lavandero, S., Kruse, C., and Machens, H. G. (2009). The use of glandular-derived stem cells to improve vascularization in scaffold-mediated dermal regeneration. Biomaterials 30, 5918-5926.

Engler, A. J., Sen, S., Sweeney, H. L., and Discher, D. E. (2006). Matrix elasticity directs stem cell lineage specification. Cell 126, 677-689.
Gerdes, J., Schwab, U., Lemke, H., and Stein, H. (1983). Production of a mouse monoclonal antibody reactive with a human nuclear antigen associated with cell proliferation. Int. J. Cancer 31, 13-20.

Gorjup, E., Danner, S., Rotter, N., Habermann, J., Brassat, U., Brummendorf, T. H., Wien, S., Meyerhans, A., Wollenberg, B., Kruse, C., and Von Briesen, H. (2009). Glandular tissue from human pancreas and salivary gland yields similar stem cell populations. Eur. J. Cell Biol. 88, 409-421.

He, W., McConnell, G. C., and Bellamkonda, R. V. (2006). Nanoscale laminin coating modulates cortical scarring response around implanted silicon microelectrode arrays. J. Neural Eng. 3, 316-326.

Hemm, S., and Wardell, K. (2010). Stereotactic implantation of deep brain stimulation electrodes: a review of technical systems, methods and emerging tools. Med. Biol. Eng. Comput. 48, 611-624.

Itosaka, H., Kuroda, S., Shichinohe, H., Yasuda, H., Yano, S., Kamei, S., Kawamura, R., Hida, K., and Iwasaki, Y. (2009). Fibrin matrix provides a suitable scaffold for bone marrow stromal cells transplanted into injured spinal cord: a novel material for CNS tissue engineering. $\mathrm{Neu}$ ropathology 29, 248-257.

have chosen this type of hydrogel amongst other reasons due to its biodegradability so it can be metabolized in vivo, associated with reduction of hydrogel thickness. We will investigate in further in vivo studies if our working hypothesis suffices for a tight device-tissue-connection.

To emphasize finally, we were able to confirm in vitro the suitability of adult stem cells as viable cellular interface for polymeric neuronal implants. We cultured adult stem cells on different processed polyimides without changes of their survival, growth and differentiation potential. Furthermore we ensured that this advantageous but sensitive modification can be brought to the target tissue without adverse effects by protecting it with fibrin. Prospectively these results should be validated in an animal model simulating a clinical setting.

\section{ACKNOWLEDGMENTS}

This work was supported by the Graduate School for Computing in Medicine and Life Sciences funded by Germany's Excellence Initiative [DFG GSC 235/1]. This study was additionally funded by the German Research Ministry project ("BiCIRTS"-13N9190), grants of the European Union [European Fund for regional development (EFRE)], and by the Business Development and Technology Transfer Corporation of Schleswig-Holstein (WTSH). We thank for the kind support Peter Detemple (IMM, Mainz, Germany) and LutzMartin Buchmann (Fraunhofer ISIT, Itzehoe, Germany) as well as Anja Rasch (Norgenta, Hamburg, Germany).

Jankovic, J., and Aguilar, L. G. (2008). Current approaches to the treatment of Parkinson's disease. Neuropsychiatr. Dis. Treat. 4, 743-757.

Johnson, P. J., Parker, S. R., and Sakiyama-Elbert, S. E. (2009a). Fibrin-based tissue engineering scaffolds enhance neural fiber sprouting and delay the accumulation of reactive astrocytes at the lesion in a subacute model of spinal cord injury. J. Biomed. Mater. Res. A 92, 152-163.

Johnson, P. J., Parker, S. R., and Sakiyama-Elbert, S. E. (2009b). Controlled release of neurotrophin-3 from fibrin-based tissue engineering scaffolds enhances neural fiber sprouting following subacute spinal cord injury. Biotechnol. Bioeng. 104, 1207-1214.

Kruse, C., Kajahn, J., Petschnik, A. E., Maass, A., Klink, E., Rapoport, D. H., and Wedel, T. (2006). Adult pancreatic stem/progenitor cells spontaneously differentiate in vitro into multiple cell lineages and form teratoma-like structures. Ann. Anat. 188, 503-517.

Larson, P. S. (2008). Deep brain stimulation for psychiatric disorders. $\mathrm{Neu}$ rotherapeutics 5, 50-58.

Leach, J., Achyuta, A. K. H., and Murthy, S. K. (2010). Bridging the divide between neuroprosthetic design, tissue engineering and neurobiology. Front. Neuroeng. 2:18. doi:10.3389/neuro.16.018.2009

Martens, H., Toader, E., Decre, M. M. J., Anderson, D. J., Vetter, R., Kipke, D. R., Baker, K. B., Johnson, M. D., and Vitek, J. L. (2010). Spatial steering of deep brain stimulation volumes using a novel lead design. Clin. Neurophysiol. 122, 558-566.

McConnell, G. C., Rees, H. D., Levey, A. I., Gutekunst, C. A., Gross, R. E., and Bellamkonda, R. V. (2009a). Implanted neural electrodes cause chronic, local inflammation that is correlated with local neurodegeneration. J. Neural Eng. 6, 56003.

McConnell, G. C., Butera, R. J., and Bellamkonda, R. V. (2009b). Bioimpedance modeling to monitor astrocytic response to chronically implanted electrodes. J. Neural Eng. 6, 055005

Mercanzini, A., Colin, P., Bensadoun, J. C., Bertsch, A., and Renaud, P. (2009). In vivo electrical impedance spectroscopy of tissue reaction to microelectrode arrays. IEEE Trans. Biomed. Eng. 56, 1909-1918.

Moss, J., Ryder, T., Aziz, T. Z., Graeber, M. B., and Bain, P. G. (2004). Electron microscopy of tissue adherent to explanted electrodes in dystonia and Parkinson's disease. Brain 127, 2755-2763. 
Petschnik, A. E., Klatte, J. E., Evers, L. H., Kruse, C., Paus, R., and Danner, S. (2009). Phenotypic indications that human sweat glands are a rich source of nestin-positive stem cell populations. Br. J. Dermatol. 162 , 380-383.

Polikov, V. S., Tresco, P. A., and Reichert, W. M. (2005). Response of brain tissue to chronically implanted neural electrodes. J. Neurosci. Methods 148, $1-18$.

Purcell, E. K., Seymour, J. P., Yandamuri, S., and Kipke, D. R. (2009). In vivo evaluation of a neural stem cellseeded prosthesis. J. Neural Eng. 6, 026005.

Rao, S. S., and Winter, J. O. (2009). Adhesion molecule-modified biomaterials for neural tissue engineering. Front. Neuroeng. 2:6. doi:10.3389/neuro.16.006.2009

Rapoport, D. H., Danner, S., and Kruse, C. (2009a). Glandular stem cells are a promising source for much more than beta-cell replacement. Ann. Anat. 191, 62-69.

Rapoport, D. H., Schicktanz, S., Gurleyik, E., Zuhlke, C., and Kruse, C. (2009b). Isolation and in vitro cultivation turns cells from exocrine human pancreas into multipotent stem-cells. Ann. Anat. 191, 446-458.

Rasche, D., Rinaldi, P. C., Young, R. F., and Tronnier, V. M. (2006). Deep brain stimulation for the treatment of various chronic pain syndromes. Neurosurg. Focus 21, E8.

Richter, A., Kruse, C., Hofmann, U., and Danner, S. (2010). Applying controlled shear stress on cell covered microprobes. Biomed. Tech. 55, 4.

Rotter, N., Oder, J., Schlenke, P., Lindner, U., Bohrnsen, F., Kramer, J., Rohwedel, J., Huss, R., Brandau, S., Wollenberg, B., and Lang, S. (2008). Isolation and characterization of adult stem cells from human salivary glands. Stem Cells Dev. 17, 509-518.

Rousche, P. J., Pellinen, D. S., Pivin, D. P. Jr., Williams, J. C., Vetter, R. J., and Kipke, D. R. (2001). Flexible polyimide-based intracortical electrode arrays with bioactive capability. IEEE Trans. Biomed. Eng. 48, 361-371.

Salem, H., Ciba, P., Rapoport, D. H., Egana, J. T., Reithmayer, K., Kadry, M., Machens, H. G., and Kruse, C. (2009). The influence of pancreasderived stem cells on scaffold based skin regeneration. Biomaterials 30 , 789-796.

Seymour, J. P., and Kipke, D. R. (2007). Neural probe design for reduced tissue encapsulation in CNS. Biomaterials 28, 3594-3607.

Spataro, L., Dilgen, J., Retterer, S., Spence, A. J., Isaacson, M., Turner,
J. N., and Shain, W. (2005). Dexamethasone treatment reduces astroglia responses to inserted neuroprosthetic devices in rat neocortex. Exp. Neurol. 194, 289-300.

Wiese, C., Rolletschek, A., Kania, G., Blyszczuk, P., Tarasov, K. V., Tarasova, Y., Wersto, R. P., Boheler, K. R., and Wobus, A. M. (2004). Nestin expression - a property of multilineage progenitor cells? Cell. Mol. Life Sci. 61, 2510-2522.

Williams, D. F. (2008). On the mechanisms of biocompatibility. Biomaterials 29, 2941-2953.

Williams, J. C., Rennaker, R. L., and Kipke, D. R. (1999). Longterm neural recording characteristics of wire microelectrode arrays implanted in cerebral cortex. Brain Res. Brain Res. Protoc. 4, 303-313.

Yasuda, H., Kuroda, S., Shichinohe, H., Kamei, S., Kawamura, R. and Iwasaki, Y. (2009). Effect of biodegradable fibrin scaffold on survival, migration, and differentiation of transplanted bone marrow stromal cells after cortical injury in rats. J. Neurosurg. 112, 336-344.

Yu, X., Dillon, G. P., and Bellamkonda, R. B. (1999). A laminin and nerve growth factor-laden threedimensional scaffold for enhanced neurite extension. Tissue Eng. 5, 291-304.
Zhong, Y., and Bellamkonda, R. V. (2008). Biomaterials for the central nervous system. J. R. Soc. Interface 5 , 957-975.

Conflict of Interest Statement: The authors declare that the research was conducted in the absence of any commercial or financial relationships that could be construed as a potential conflict of interest.

Received: 07 June 2011; paper pending published: 12 August 2011; accepted: 07 September 2011; published online: 10 October 2011.

Citation: Richter A, Kruse C, Moser A, Hofmann UG and Danner S (2011) Cellular modulation of polymeric device surfaces: promise of adult stem cells for neuro-prosthetics. Front. Neurosci. 5:114. doi: 10.3389/fnins.2011.00114

This article was submitted to Frontiers in Neuroprosthetics, a specialty of Frontiers in Neuroscience.

Copyright $\odot 2011$ Richter, Kruse, Moser, Hofmann and Danner. This is an openaccess article subject to a non-exclusive license between the authors and Frontiers Media SA, which permits use, distribution and reproduction in other forums, provided the original authors and source are credited and other Frontiers conditions are complied with. 